\title{
Condition-Based Maintenance Models for Stone Claddings
}

\author{
Cláudia Ferreira ${ }^{1}$, Ana Silva ${ }^{1}$, Jorge de Brito ${ }^{1}$ and Luís C. Neves ${ }^{2}$ \\ ${ }^{1}$ CERIS, Instituto Superior Técnico, University of Lisbon, Av. Rovisco Pais, Lisbon, Portugal, \\ claudiaarferreira@tecnico.ulisboa.pt, anasilva931@msn.com,jb@civil.ist.utl.pt \\ ${ }^{2}$ Resilience Engineering Research Group, University of Nottingham, Nottingham, United Kingdom, \\ luis.neves@nottingham.ac.uk
}

\begin{abstract}
Most of the maintenance actions in the construction sector are still reactive and sporadic, based on subjective criteria. Usually, maintenance actions are performed when the building already presents unacceptable degradation levels, and the reactive maintenance actions carried out only correct the anomalies observed, not dealing with the causes, thus leading to additional costs and risks for owners. This study establishes a condition-based maintenance model applied to natural stone claddings. This model has a stochastic nature, in order to deal with the complexity of the degradation phenomena. For that purpose, Petri nets are used, providing an efficient tool to model the deterioration process and the maintenance decisions considered for stone claddings. The maintenance model is a full life-cycle model that encompasses: (i) the stochastic assessment of the degradation condition of the stone claddings, and their expected service life; (ii) and the inspections, maintenance and renewal processes. In this study, three maintenance strategies are considered: (i) major intervention only; (ii) combination of minor and major interventions; and (iii) combination of cleaning operations, minor and major interventions. The impact of the different maintenance strategies in the future performance and remaining service life of stone claddings is analysed, also evaluating the economic impact of each maintenance plan.
\end{abstract}

Keywords: Degradation, Service Life, Maintenance, Natural Stone Claddings, Petri Net.

\section{Introduction}

The awareness of the building managers and/or owners about the need to maintain their assets more efficiently has increased over the last years (Silva and de Brito, 2019). The maintenance of buildings' envelope elements is not yet a first concern (Forster and Kayan, 2009; Thomsen and van der Flier, 2011). Usually, maintenance actions are carried out when the buildings' envelope elements are already severely degraded, or reactive maintenance actions are performed to react to failures and do not deal with the causes (Mobley, 2014), thus leading to additional costs and risks (Dann and Cantell, 2005). Furthermore, regular inspections to assess the condition and maintenance actions on these elements are not mandatory (Shohet et al., 2002). The definition of the end of their service life is subjective, based on the outward appearance, owners/users' demands and the funds available for maintenance works (Emídio et al., 2014).

Therefore, the development of more efficient methodologies for the implementation of strategic planning of maintenance actions is important (Lacasse and Vanier, 1996; Aikivuori, 1999). The existing tools have several limitations (Ashworth, 1996; Sherwin, 2000; Forster and Kayan, 2009): scarce data about maintenance protocols; poor or non-existent implementation of strategic procedures; lack of information regarding durability and performance of buildings; insufficiently accuracy of the existing maintenance policies; and lack of infor- 
mation about global costs associated with repairs. This information is extremely relevant to managers, insurers, owners or users, and allows the adoption of more sustainable and durable solutions at the design stage and for the definition and implementation of reliable maintenance policies, enabling a more rational management of the building stock.

The main purpose of this study is the development and implementation of a conditionbased maintenance model for Natural Stone Claddings (NSC). The proposed model is implemented using a stochastic Petri Nets (PN) framework. The case study selected is composed of 203 NSC, located in Portugal. The sample was established based on the diagnosis of the degradation condition of these claddings in-service performance, through in situ visual inspection. In the methodology developed, three maintenance strategies are assessed: (i) major intervention only; (ii) combination of minor and major interventions; and (iii) combination of cleaning operations, minor and major interventions. The first one represents the most common solution adopted by buildings' owners. The other two are analysed in order to evaluate the impact of the different alternatives in the claddings' service life and in the whole-life maintenance costs, for the period under analysis.

\section{Petri Nets (PN)}

PN are a mathematical and graphical modelling tool, introduced by Carl A. Petri in 1962 (Petri, 1962), suitable for description of systems whose dynamics are characterized as being concurrent, asynchronous, distributed, parallel, nondeterministic, and/or stochastic (Murata, 1989).

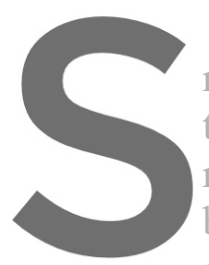

In a few words, PN

nodes, places (represen tions (representing an

rected arcs. An arc connects

black dots) are stored in places and its distributir

current state of the system. Transitions are responsible for the
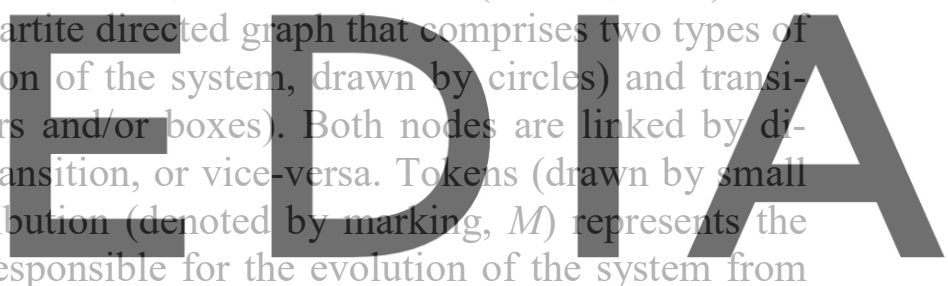

one state to another (movement of the tokens between places). A transition is enabled to fire

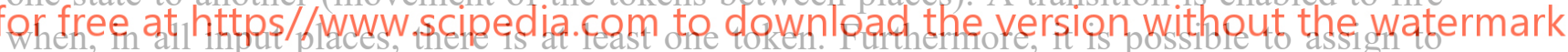

each transition a firing rate that can be deterministic or modelled by any probabilistic distribu-

tion. Figure 1 shows an example of PN composed of three piaces $\left(p_{1}, p_{2}, p_{3}\right)$ and one transition $\left(t_{1}\right)$. In this example, the transition is not enabled to fire since place $p_{2}$ is empty.

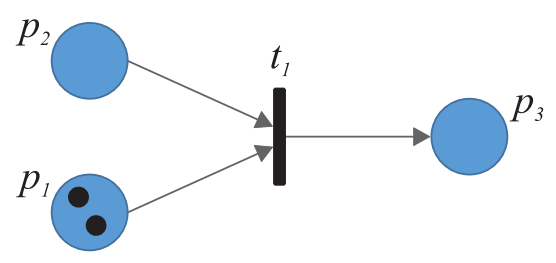

Figure 1. Example of a Petri net.

\section{Maintenance Model}

The maintenance model, based on the Petri nets methodology, proposed to assess the performance of NSC, is presented in Figure 2. Below, the meaning of each part of the model is briefly described. More information about the model can be seen in Ferreira et al. (2019). 


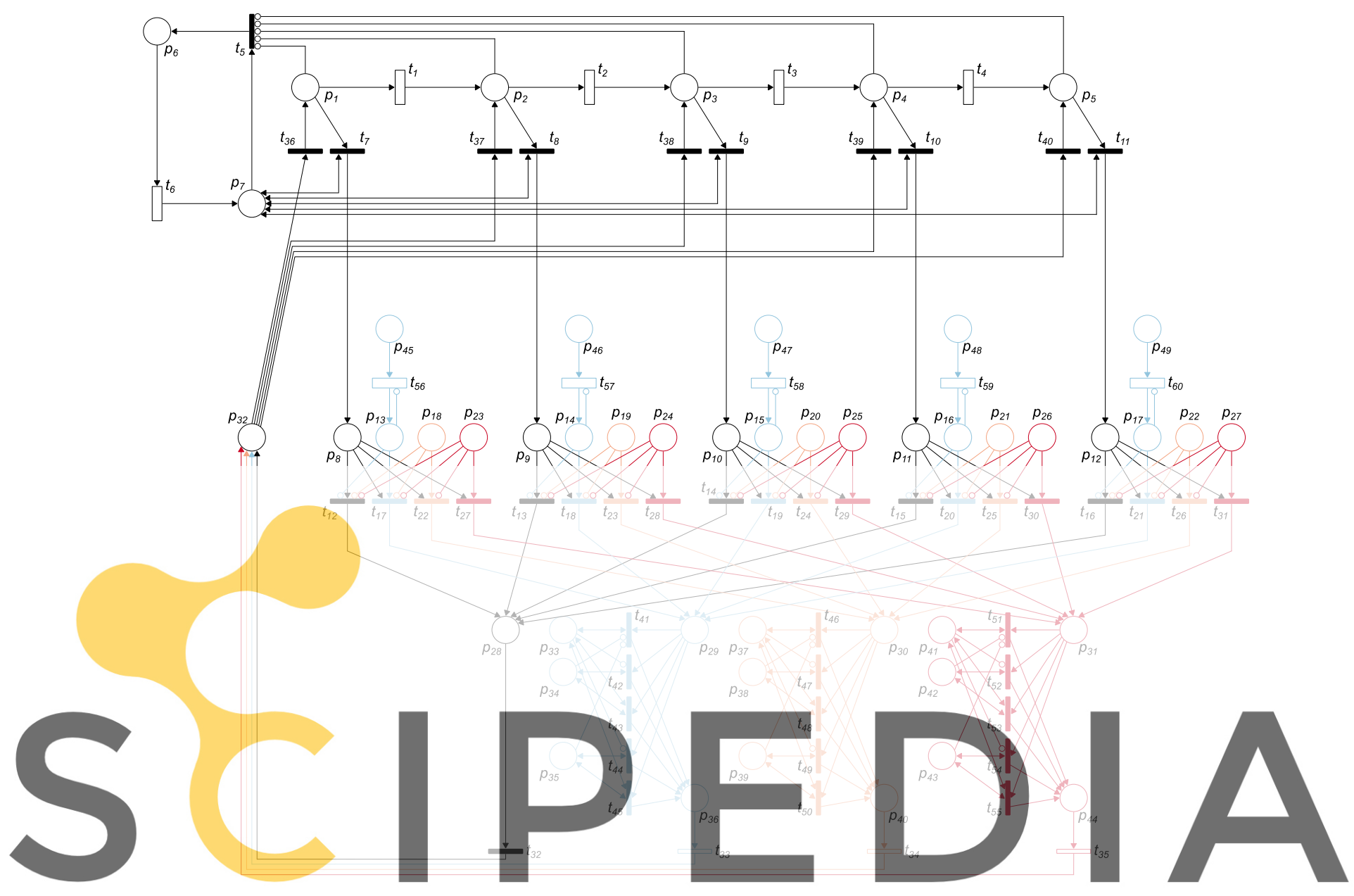

Figure 2. Petri net scheme of the maintenance model proposed.

Register for free at https//www.scipedia.com to download the version without the watermark The maintenance model implemented is a condition-based model, where it is assumed that maintenance actions are planned after the condition has been assessed through inspections. In other words, the need for a maintenance action is assessed during inspection, and then, according to the current observed degradation condition of the component under analysis, a decision on intervening is made. The model can be divided in several main parts: (i) degradation process; (ii) inspection process; (iii) maintenance process; (iv) modelling of the maintenance actions; and (v) periodicity of the cleaning operations.

In Figure 2, the degradation process is depicted by five places ( $p_{1}$ to $\left.p_{5}\right)$ and four timed transitions $\left(t_{1}\right.$ to $\left.t_{4}\right)$. Each place denotes a condition level defined in the classification system adopted, where places $p_{1}$ and $p_{5}$ represent, respectively, the best and worst condition level of the classification system. The timed transitions, located between places, model the transition times of moving between different condition levels. The transition times are samples from the appropriate distributions that can be obtained by studying the degradation characteristics of the NSC (Le and Andrews, 2016). The classification system adopted (Table 1), proposed by Silva et al. (2011), is based on visual inspections of the extent of the cladding area affected by the different anomalies. The severity of degradation index, $S_{w}$, is defined with the intent of expressing the overall degradation condition of these claddings, and is given by the ratio between the area affected by the defects 
observed in a NSC, weighted according to their severity, and a reference area equivalent to the total cladding area with the highest possible level of degradation (Equation 1) (Silva et al., 2016).

$$
S_{w}=\frac{\sum\left(A_{n} \times k_{n} \times k_{a, n}\right)}{A \times \sum k}
$$

where $S_{w}$ represents the severity of degradation (in \%), $k$ the multiplying factor corresponding to the highest degradation condition level of the cladded area $A, A_{n}$ the cladding area affected by any defect $n$ (in $\mathrm{m}^{2}$ ), $k_{n}$ the multiplication factor for the defect $n$, in terms of their degradation condition, and $A$ the total area of the NSC (in $\mathrm{m}^{2}$ ). More information about the classification of defects and the determination of $S_{w}$ can be found in Silva et al. (2016).

Table 1. Degradation conditions for natural stone cladding.

\begin{tabular}{cc}
\hline Degradation condition & Severity of degradation \\
\hline Condition A: No visible degradation & $S_{w} \leq 1 \%$ \\
\hline Condition B: Good & $1 \%<S_{w} \leq 8 \%$ \\
\hline Condition C: Slight degradation & $8 \%<S_{w} \leq 20 \%$ \\
\hline Condition D: Moderate degradation & $20 \%<S_{w} \leq 45 \%$ \\
\hline Condition E: Generalized degradation & $S_{w} \geq 45 \%$ \\
\hline
\end{tabular}

The inspection process is modelled through the cycle formed by nodes: $p_{6}-t_{6}-p_{7}-t_{5}-p_{6}$ (Andrews, 2013). A token in place $p_{6}$ means that an inspection should not be performed at that time and enables transitions $t_{6}$ that manages the time interva token in place $p_{7}$ means that it is time to perform an inspection, it enables one of the trar the true condition of the that the true condition of the clad which causes the token in place $p_{7}$ to return to place $p_{6}$ to wait for the next inspection.

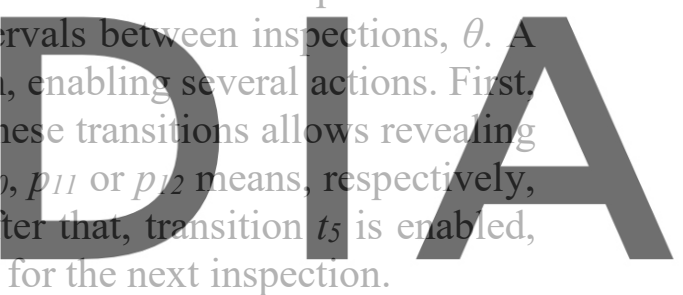

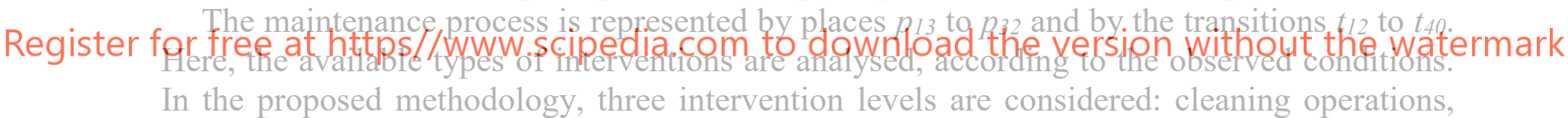
minor and major interventions. This information is introduced in the model through places $p_{13}$ to $p_{27}$. Tokens in places $p_{13}$ to $p_{17}$ mean cleaning operations must be done, in $p_{18}$ to $p_{22}$ that a minor intervention is needed, and in $p_{23}$ to $p_{27}$ that a major intervention is required. If there is no token in these places, no interventions are performed. Otherwise, if, for the same condition, there is more than one type of intervention available, the highest impact intervention is performed. A token in places $p_{28}, p_{29}, p_{30}$ or $p_{31}$ indicates, respectively, that no intervention, cleaning operations, minor or major interventions are required. After that, the token returns to the degradation process by place $p_{32}$ and transitions $t_{36}$ to $t_{40}$.

In Figure 2, the cleaning operations is modelled by places $p_{33}$ to $p_{36}$ and by transitions $t_{41}$ to $t_{45}$. The marking of places $p_{33}, p_{34}$ or $p_{35}$ means, respectively, that the cleaning operation, when applied, has the effect of improving the condition, supressing the degradation process or reducing the deterioration rate. Then, depending on the impact of the cleaning operation on the cladding, only one transition can be fired. For the minor (places $p_{37}$ to $p_{40}$ and transitions $t_{46}$ to $t_{50}$ ) and major (places $p_{41}$ to $p_{44}$ and transitions $t_{51}$ to $t_{55}$ ) interventions, the same methodology is implemented. 
In this model, the periodicity of the cleaning operations is considered through places $p_{45}$ to $p_{49}$ and timed transitions $t_{56}$ to $t_{60}$. The introduction of the information that this intervention is available in a given condition level is performed by placing tokens in places $p_{45}$ to $p_{49}$. Transitions $t_{56}$ to $t_{60}$ are associated with a delay that allows, at the end of $\theta_{\text {clean }}$ time units, the tokens present in places $p_{45}$ to $p_{49}$ to be returned to places $p_{13}$ to $p_{17}$, allowing cleaning operations to be performed at the next inspection time, if the imposed constrains are complied with.

\section{Case Study: Natural Stone Claddings}

The case study under examination is composed of 203 NSC directly adhered to the substrate, located in Portugal. The degradation condition of each cladding in the data set was analysed based on in situ visual inspections. The methodology described, was applied to analyse the degradation of NSC over their lifetime. A 150-year time horizon was considered and the cladding is expected to be in perfect conditions at the beginning of the analysis (in condition A).

\subsection{Probabilistic Analysis of the Deterioration Process}

To find the best distribution that describes the degradation characteristics of the NSC, the degradation process (without maintenance) is applied to the complete sample. Table 2 shows the optimized estimation parameters obtained for the three distribution functions (Exponential, Weibull and Lognormal) analysed, in terms of mean time in each degradation condition and standard deviation, as well as the respective log-likelihood value.
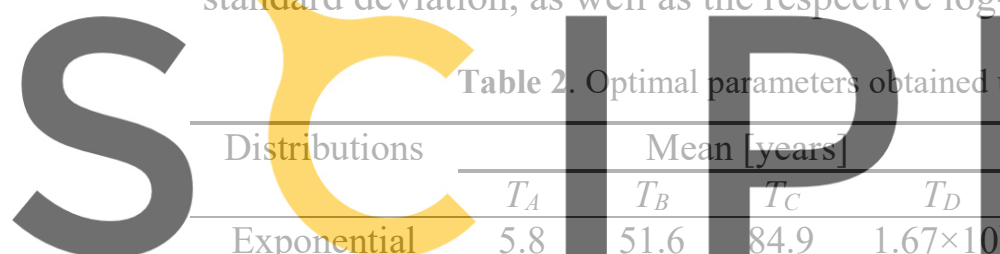

Exponential

5.8

51.6

$84.9+1.67 \times 10^{7}$

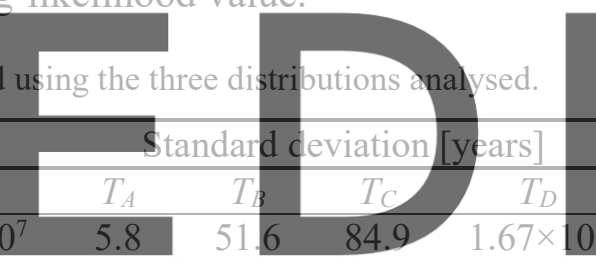

7.0

10.3

2.4



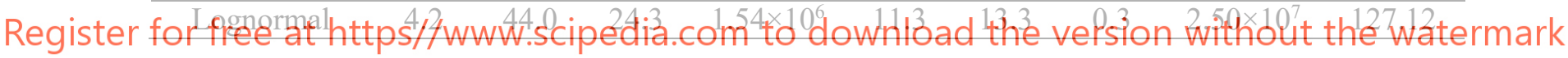

The parameters of the probability density function are fitted to the historic database based on the concept of maximum likelihood (Kalbfleisch and Lawless, 1985). The likelihood, $L$, is defined as the predicted probability of occurrence of the observed transitions (Equation 2).

$$
L=\prod \prod p_{i j} \Rightarrow \log L=\sum \sum p_{i j}
$$

where $p_{i j}$ is the probability of transition from deterioration condition $i$ to $j$.

The results allow concluding that two-parameter distributions (Weibull and Lognormal) show a better adjustment to the data set than the Exponential distribution. Since Weibull distribution is the one that shows a minor $\log$-likelihood, $\log L$, value and, consequently, a better fit to the historical data, this distribution is chosen to sample the transitions times that specify the movement between different conditions levels.

\subsection{Maintenance Strategies and Costs}

In this study, three Maintenance Strategies (MS) are considered: (i) major intervention only (MS1); (ii) combination of minor and major interventions (MS2); and (iii) combination of clean- 
ing operations, minor and major interventions (MS3). The different strategies were defined based on previous works and on experts' judgement. Table 3 presents the fixed costs, application zones and impacts of the different types of interventions considered. It was assumed that an Alpinina marble cladding, directly adhered to the substrate, was used. For example, a cleaning operation of NSC has a cost of $31.37 € / \mathrm{m}^{2}$, is applied in condition B and improves it to condition A with a probability of $15 \%$ or causes no significant improvement with a probability of $85 \%$. Regarding the inspections, a deterministic periodicity of 5 years is considered.

Table 3. Fixed costs, application zones and impacts of the different types of interventions considered.

\begin{tabular}{cccccc}
\hline Interventions & Cost $\left[€ / \mathrm{m}^{2}\right]$ & \multirow{2}{*}{\begin{tabular}{c} 
Application \\
\cline { 4 - 6 }
\end{tabular}} & & \multicolumn{3}{c}{ Impact of the maintenance actions [\%] } \\
\cline { 5 - 6 } Inspections & 1.03 & All & $P_{A}$ & $P_{B}$ & $P_{C}$ \\
\hline Cleaning operations & 31.37 & $\mathrm{~B}$ & 15.0 & 85.0 & - \\
\hline Minor interventions & 68.80 & $\mathrm{C}$ & - & 80.4 & - \\
\hline Major interventions & 149.51 & $\mathrm{D}, \mathrm{E}$ & 100.0 & - & - \\
\hline
\end{tabular}

\subsection{Comparison of the Different Maintenance Strategies}

Figure 3 compares the degradation curves obtained for the three MS and for the situation without interventions. The results show that the proposed MS have a significant impact on the mean severity of degradation. For all MS, the mean severity of degradation is less than $8 \%$

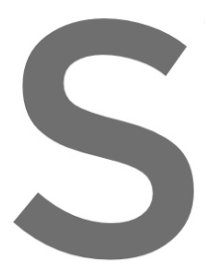
(condition B), with MS
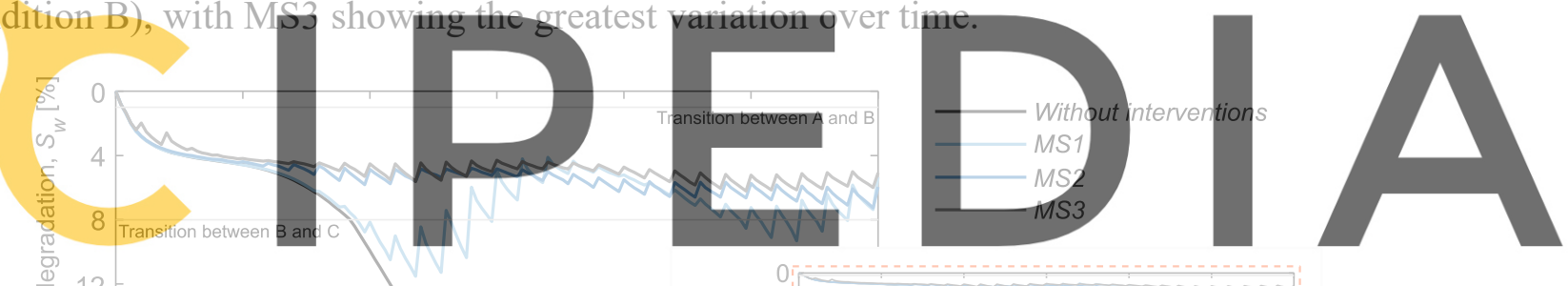

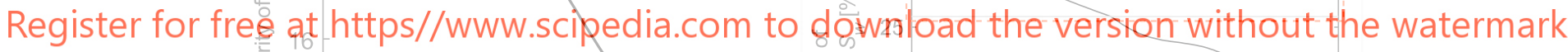
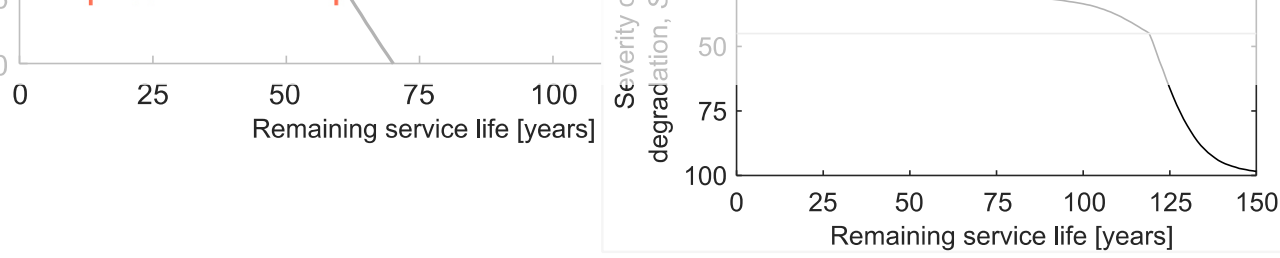

Figure 3. Comparison of the degradation curves over time for all maintenance strategies.

On the other hand, if the cumulative costs are analysed (Figure 4), MS2 has the lowest cumulative costs when the discount rate is $0 \%$ (Figure 4a) and MS1 for a discount rate of $6 \%$ (Figure $4 \mathrm{~b}$ ). For both rates, MS3 is the one with the highest cumulative maintenance costs.

The discount rate is used to balance the initial investment costs against the future maintenance cost. Since this parameter is difficult to predict, two extreme values were considered to assess the sensitivity of the results. Its choice is mainly a political decision, and the value usually ranges between $4 \%$ and $6 \%$ (van Noortwijk and Frangopol, 2004).

Finally, the number of interventions and the remaining service life for the three MS are analysed (Figure 5). According to Silva et al. (2016), the end of the service life of a NSC varies 
between 68 and 90 years. The end of the service life occurs when a major intervention is performed. If the first major intervention is analysed (Figure 5a), for MS1, in year 85 there is a probability of $90 \%$ of NSC having been replaced at least once. The value agrees with the range suggested by Silva et al. (2016). On the other hand, for MS2, in year 150 there is only a $58 \%$ probability that the NSC has been replaced at least once, and, for EM3, this percentage in the same year is reduced to $28 \%$. From this it results that combining major intervention with other intervention works, such as minor interventions and cleaning operations, increases the service life of NSC. Similar observations can be made for the minor intervention (Figure $5 b)$. Considering cleaning operations in MS3 postpones the need of a minor intervention.

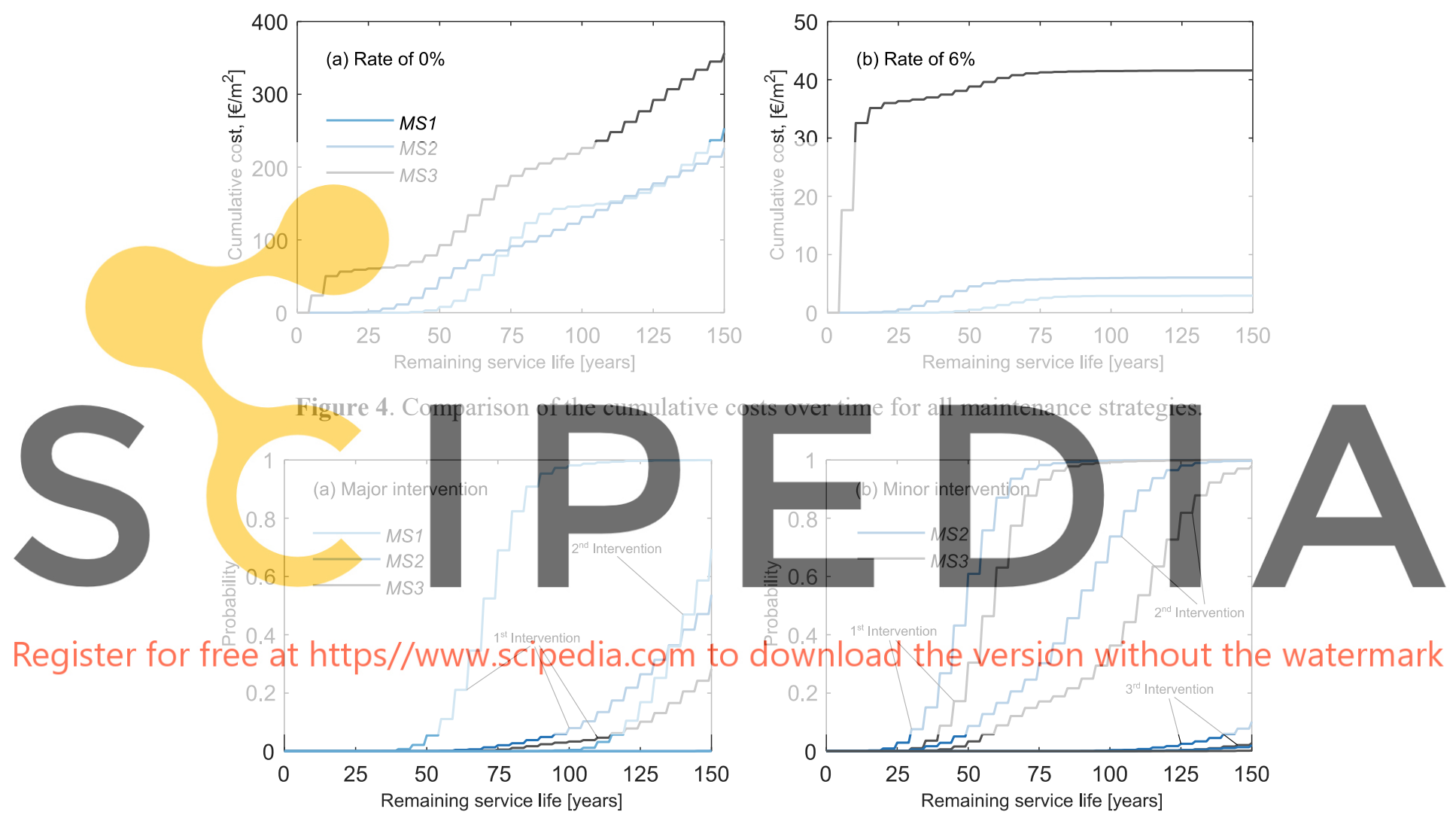

Figure 5. Comparison of the cumulative distribution function of the number of interventions.

\section{Conclusions}

In this study, a maintenance model, based on the PN formalism, is proposed to analyse the impact of different MS to control deterioration conditions of NSC. In the proposal methodology, three MS are analysed. These are composed by three different intervention levels: cleaning operations, minor and major interventions. The results reveal that: MS1 presents the lowest cumulative cost for a discount rate of $6 \%$. In MS1, only replacement of the cladding is considered, which does not allow improving the condition over time, as well as its service life. MS3 shows the best degradation results but leads to the highest cumulative costs over the claddings' lifetime. MS2 seems to be the most rational and adequate solution, according to the model's assumptions, since it allows increasing the service life and the cumulative costs are competitive when compared with those of 
MS1. However, in the end, the choice of the best MS is always assumed by the building managers and/or owners and depends on their budgets and target condition for the cladding.

\section{Acknowledgements}

The authors acknowledge the support of the Foundation for Science and Technology, CERIS Research Centre and Instituto Superior Técnico.

\section{ORCID}

Ana Silva: http://orcid.org/0000-0001-6715-474X

Jorge de Brito: http://orcid.org/0000-0001-6766-2736

Luís C. Neves: http://orcid.org/0000-0001-5034-8417

\section{References}

Aikivuori, A.M. (1999). Critical loss of performance - What fails before durability. Performance Based Methods for Service Life Prediction Reports compiled, CIB W80.

Andrews, J. (2013). A modelling approach to railway track asset management. Journal of Rail and Rapid Transit, 227(1), 56-73.

Ashworth, A. (1996). Estimating the life expectancies of building components in life-cycle costing calculations. Struetural Survey, 14(2), 4-8.

Dann, N. and Cantell, T. (2005). Maintenance: from philosophy to practice. Journal of Architectural Conservation, 11(1), $42-54$.

Emídio, F., de Brito, J., Gaspar, P. L. and Silva, A. (2014). Application of the factor method to the estimation of the service life of natural stone cladding. Construction and Building Materials, 66, 484-493.

Ferreira, C., Neves, L.C., Silya, A. and de Brito, (2019). Stochastic maintenance models for ceramic claddings.
Structure and Infrastructure Engineering, $6(2), 247-265$.
Forster, A.M. and Kayan, B. (2009). Maintenance for historic buildings: a current perspective. Structural Surver,
27(3), 210-229.
Kalbfleisch, J.D. and Lawless, J.F. (1985). The analysis of the panel data under a Markoy assumption. Journot of
the American Statistical Association.80(392), 863-871.

Lacasse, M.A. and Vanier, D.J. (1996). A review of service life and durability issues. In Proceedings of the VII

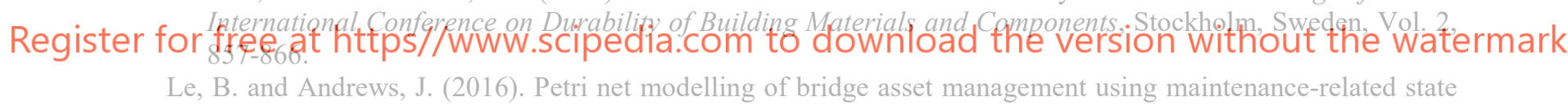
conditions. Structure and Infrastructure Engineering, 12(6), 730-751.

Mobley, R.K. (2014). Maintenance Engineering Handbook. New York, NY: MaGraw-Hill Education.

Murata, T. (1989). Petri nets: Properties analysis and applications. Proceedings of the IEEE, 77(4), 541-580.

Petri, C.A. (1962). Kommunikation mit automaten (in German), $\mathrm{PhD}$ Thesis, Institut für Instrumentelle Mathematik an der Universität Bonn, Bonn, Germany.

Sherwin, D. (2000). A review of overall models for maintenance management. Journal of Quality in Maintenance Engineering, 6(3), 138-164.

Shohet, I.M., Puterman, M. and Gilboa, E. (2002). Deterioration patterns of building cladding components for maintenance management. Construction Management and Economics, 20(4), 305-314.

Silva, A., de Brito, J. and Gaspar, P.L. (2011). Service life prediction model applied to natural stone wall claddings (directly adhered to the substrate). Construction and Building Materials, 25(9), 3674-3684.

Silva, A., de Brito, J. and Gaspar, P.L. (2016). Methodologies for service life prediction of buildings: With a focus on façade claddings. Switzerland: Springer International Publishing.

Silva, A. and de Brito, J. (2019). Do we need a buildings' inspection, diagnosis and service life prediction software?. Journal of Building Engineering, 22, 335-348.

Thomsen, A. and van der Flier, K. (2011). Understanding obsolescence: a conceptual model for buildings. Building Research \& Information, 39(4), 352-362. doi: 10.1080/09613218.2011.576328

van Noortwijk, J.M. and Frangopol, D.M. (2004). Two probabilistic life-cycle maintenance models for deteriorating civil infrastructures. Probabilistic Engineering Mechanics, 19(4), 345-359. 